\title{
NANOG Attenuates Hair Follicle-Derived Mesenchymal Stem Cell Senescence by Upregulating PBX1 and Activating AKT Signaling
}

\author{
Feilin Liu $(\mathbb{D})$, ${ }^{1,2}$ Jiahong Shi $\left(\mathbb{D},{ }^{1,3}\right.$ Yingyao Zhang $\mathbb{D}^{1},{ }^{1}$ Aobo Lian $\mathbb{D D}^{1},{ }^{1}$ Xing Han $\left(\mathbb{D},{ }^{1}\right.$ \\ Kuiyang Zuo $\mathbb{D}^{1},{ }^{1}$ Mingsheng Liu $\mathbb{D}^{1},{ }^{1}$ Tong Zheng $\mathbb{D}^{1},{ }^{1}$ Fei Zou $\mathbb{D}^{1},{ }^{1}$ Xiaomei Liu ${ }^{(D)}{ }^{1}$ \\ Minghua Jin $\oplus,{ }^{1}$ Ying Mu $\odot,{ }^{4}$ Gang Li $\odot{ }^{5}$ Guanfang Su $\odot{ }^{2}$ and Jinyu Liu $\oplus^{1}$ \\ ${ }^{1}$ Department of Toxicology, School of Public Health, Jilin University, Changchun, China \\ ${ }^{2}$ Department of Ophthalmology, The Second Hospital of Jilin University, Changchun, China \\ ${ }^{3}$ Department of Ultrasound, The China-Japan Union Hospital of Jilin University, Changchun, China \\ ${ }^{4}$ Research Center for Analytical Instrumentation, Institute of Cyber-Systems and Control, State Key Laboratory of Industrial \\ Control Technology, Zhejiang University, Hangzhou, China \\ ${ }^{5}$ Department of Orthopaedics \& Traumatology, Li Ka Shing Institute of Health Sciences, Chinese University of Hong Kong, Prince of \\ Wales Hospital, Shatin, Hong Kong, China \\ Correspondence should be addressed to Guanfang Su; sugf2012@163.com and Jinyu Liu; jy_liu@jlu.edu.cn
}

Received 9 May 2019; Revised 24 September 2019; Accepted 12 October 2019; Published 4 December 2019

Academic Editor: Gianluca Carnevale

Copyright ( 2019 Feilin Liu et al. This is an open access article distributed under the Creative Commons Attribution License, which permits unrestricted use, distribution, and reproduction in any medium, provided the original work is properly cited.

\begin{abstract}
Stem cells derived from elderly donors or harvested by repeated subculture exhibit a marked decrease in proliferative capacity and multipotency, which not only compromises their therapeutic potential but also raises safety concerns for regenerative medicine. NANOG-a well-known core transcription factor-plays an important role in maintaining the self-renewal and pluripotency of stem cells. Unfortunately, the mechanism that NANOG delays mesenchymal stem cell (MSC) senescence is not well-known until now. In our study, we showed that both ectopic NANOG expression and PBX1 overexpression (i) significantly upregulated phosphorylated AKT (p-AKT) and PARP1; (ii) promoted cell proliferation, cell cycle progression, and osteogenesis; (iii) reduced the number of senescence-associated- $\beta$-galactosidase- (SA- $\beta$-gal-) positive cells; and (iv) downregulated the expression of p16, p53, and p21. Western blotting and dual-luciferase activity assays showed that ectopic NANOG expression significantly upregulated PBX1 expression and increased $P B X 1$ promoter activity. In contrast, $P B X 1$ knockdown by RNA interference in hair follicle- (HF-) derived MSCs that were ectopically expressing NANOG resulted in the significant downregulation of p-AKT and the upregulation of p16 and p21. Moreover, blocking AKT with the PI3K/AKT inhibitor LY294002 or knocking down AKT via RNA interference significantly decreased PBX1 expression, while increasing p16 and p21 expression and the number of SA- $\beta$ gal-positive cells. In conclusion, our findings show that NANOG delays HF-MSC senescence by upregulating PBX1 and activating AKT signaling and that a feedback loop likely exists between PBX1 and AKT signaling.
\end{abstract}

\section{Introduction}

Mesenchymal stem cells (MSCs) - which are characterized by self-renewal, multipotency, nontumorigenic properties, immune-privilege, and rich sources [1] - are considered to be an ideal resource for stem cell-based regenerative medicine. Increasing numbers of animal tests and clinical trials show that MSCs derived from various tissues, in particular, those from solid tissues [2] (e.g., bone marrow, fat, and hair follicles) and placenta exhibit tremendous therapeutic potential in alleviating cardiac infarction [3], end-stage liver failure [4], and kidney fibrosis [5], as well as in enhancing the recovery of chronic skin ulcers [6] and neuronal regeneration [7]. Hair follicle- (HF-) derived MSCs can be easily derived, highly expanded, and used as one of most popular cell sources for regenerative medicine. HF-MSCs not only take 
part in the generation of hair follicles but also contribute to hair cycle progression. The senescence of HF-MSCs will not only decrease self-renewal and multipotent capacities but also affect hair growth and regeneration.

NANOG, a homeodomain transcription factor, is expressed in the inner cell mass of embryonic stem cells (ESCs) during early embryonic development and the developing germlines of mammals. In conjunction with Oct4 and Sox2, NANOG plays an important role in maintaining stem cell pluripotency and self-renewal and in reprogramming human somatic cells into pluripotent stem cells [8]. In concert with Oct4, NANOG has been shown to facilitate self-renewal and maintain MSCs in an undifferentiated state by directly binding the Dnmt1 promoter [9]. Studies by the Andreadis' group showed that the ectopic expression of NANOG delays the culture-associated senescence of hair follicle-derived MSCs, reverses the organismal aging of bone marrow-derived MSCs (BM-MSCs), and restores impaired myogenic potential [2, 10-12]. Furthermore, Munst et al. [13] showed that NANOG enhances proliferation and suppresses replicative senescence in human primary fibroblasts by downregulating $\mathrm{p} 27^{\mathrm{KIP} 1}$. These experimental data suggest that the transcription factor NANOG plays a vital role in maintaining self-renewal and in pluripotent or multipotent differentiation in stem cells, while suppressing spontaneous senescence during stem cell culture.

PBX1, a homeodomain transcription factor from the three-amino acid loop extension family, is involved in various developmental processes such as organogenesis and hematopoiesis [14, 15]. In synergy with Hox and Meis proteins, PBX1 targets Fgf10 to regulate the development of the lung mesenchyme [16]. In addition, it actively participates in skeletal muscle differentiation by binding the Myogenin promoter [17] and regulates bone mineral density by increasing the expression of Runx2 and Osterix, while decreasing the proliferation of osteoblasts and the formation of bone nodules [18]. Moreover, PBX1 is required for the maintenance and self-renewal of hematopoietic stem cells [15]. Furthermore, PBX1 inhibits the development of adipocytes by promoting the proliferation of adipocyte progenitors postnatally [19]. Studies by Wang and colleagues [20] found an interaction loop between NANOG and PBX1. By directly binding the proximal promoter of NANOG, PBX1 upregulates the expression of endogenous NANOG protein in human ESCs, which may activate downstream cell signaling cascades [21]. Moreover, NANOG has been shown to promote $P B X 1$ transcription in both human ESCs and NIH3T3 cells $[22,23]$. However, the interaction between PBX1 and NANOG during MSC senescence has not yet been studied.

The phosphatidylinositol 3 kinase (PI3K)/protein kinase $\mathrm{B}$ (also known as AKT) pathway is considered to be a key pathway in the regulation of cell metabolism-associated processes including proliferation, DNA repair, and senescence $[24,25]$. Our previous study showed that EGF promotes the proliferation of human hair follicle-derived mesenchymal stem cells (HF-MSCs) by activating the AKT pathway [26]. Some studies suggest that AKT activation inhibits cellular senescence while reducing the expression of p16, p53, and p21. Further, Palumbo et al. [24] found that macrophage migration inhibitory factor delays human MSC senescence by activating AKT signaling. In addition, Liu et al. [27] showed that activation of the AKT pathway can enhance growth and inhibit senescence in skin-derived precursors. In contrast, Kim et al. [28] revealed that p53-induced cellular senescence is required for cooperation between p21 and AKT. Thus, there is no consistent conclusion regarding the relationship between AKT and cellular senescence. These discrepancies may result from differences in cell types or cell states. Furthermore, the signaling pathways responsible for the delay in HF-MSC senescence as a result of NANOG have not yet been reported.

However, the cellular senescence that results from organismal aging or cell culture exerts considerable effects on the quantity and quality of MSCs, which not only compromises the therapeutic potential of the MSCs but also raises safety concerns for stem cell-based regenerative medicine. As such, it is necessary to develop novel strategies for maintaining MSCs in a highly proliferative but undifferentiated state. To this end, we isolated MSCs from hair follicles and transduced them with lentiviral vectors to express NANOG or PBX1. Our research shows that the ectopic expression of NANOG promotes cell proliferation and delays HF-MSC senescence by enhancing PBX1 expression and activating downstream AKT signaling. Moreover, we uncovered a previously unknown positive feedback loop between PBX1 and AKT in these cells.

\section{Materials and Methods}

The protocols for all experiments in the present study were approved by the Institutional Review Board of the Jilin University School of Public Health.

2.1. Isolation, Cultivation, and Phenotypic Characterization of HF-MSCs. The isolation and culture of HF-MSCs were performed according to previously published methods $[26,29,30]$. Briefly, after three washes with phosphatebuffered saline (PBS), hair follicles were transferred into a 24-well culture plate, at one or two follicles per well, and cultured in Dulbecco's Modified Eagle Medium-Ham F-12 (DMEM/F-12; Gibco, Life Technologies, USA) supplemented with $10 \%$ fetal bovine serum (FBS; Gibco, USA) and $2 \mathrm{ng} / \mathrm{ml}$ of bFGF (Sino Biological Inc., China) at $37^{\circ} \mathrm{C}$ with $5 \% \mathrm{CO}_{2}$. The culture medium was changed every 3 days. After spindle-shaped cells migrated out from the hair follicles and reached $80 \%$ confluency, cells were digested with $0.25 \%$ trypsin and subsequently subcultured (Biosharp, China). Immunofluorescence assays and flow cytometry [26] were then performed to evaluate the expression of the following cell surface markers: CD105, CD90, CD73, CD44, and CD31 (eBioscience, USA).

2.2. Lentiviral Plasmid Construction and Transduction. The human NANOG coding region (Accession No. NM_ 024865) was first cloned into the pLVX-IRES-ZsGreen1 lentivirus vector (Youbio, China). 
PBX1 forward ( $5^{\prime}$-GGAGGGTTTCTCTCCCAACG- $3^{\prime}$ ) and reverse (5'-GGGAGTCTAGGACAGAGGCA- $\left.3^{\prime}\right)$ primers were synthesized by Sangon Biotech (Shanghai, China), and PBX1 cDNA was obtained by PCR amplification (PCR SuperMix Kit; TRANSGEN BIOTECH, China) according to the manufacturer's protocols. After enzymatic digestion and ligation, the human $P B X 1$ coding region (Accession No. NM_002585) was cloned into the pLVXIRES-ZsGreen1 lentiviral vector (Youbio, China).

Next, $6 \times 10^{6} 293 \mathrm{~T}$ cells (stored in the Department of Toxicology, School of Public Health, Jilin University) were seeded in a $100 \mathrm{~mm}$ cell culture dish (NEST, China) and cultured at $37^{\circ} \mathrm{C}$ with $5 \% \mathrm{CO}_{2}$ in High Glucose DMEM (Gibco, Life Technologies, USA) containing 10\% FBS (Gibco, USA) overnight. The following day, $10 \mu \mathrm{g}$ of lentivirus vector encoding NANOG or PBX1,7.5 $\mu \mathrm{g}$ of psPAX2-gag/$\mathrm{pol} / \mathrm{tat} / \mathrm{rev}$, and $2.5 \mu \mathrm{g}$ of pMD2.G-VSVG were mixed with $22 \mu \mathrm{l}$ of EndoFectin $^{\mathrm{TM}}$ Max Transfection Reagent (GeneCopoeia, USA) in $1 \mathrm{ml}$ of Opti-MEM (Gibco, Life Technologies, USA) and added onto $293 \mathrm{~T}$ cells one drop at a time. After $6 \mathrm{~h}$, the medium was replaced with fresh High Glucose DMEM containing 10\% FBS and cultured for another $36 \mathrm{~h}$. At the end of the cultivation period, the supernatant was harvested, filtered through a $0.45 \mu \mathrm{m}$ polystyrene filter (Millipore, America), and added to HFMSCs supplemented with $10 \%$ FBS, $2 \mathrm{ng} / \mathrm{ml}$ bFGF (Sino Biological Inc., China) and $10 \mu \mathrm{g} / \mathrm{ml}$ polybrene (Santa, USA). Subsequently, $72 \mathrm{~h}$ after transduction, the HF-MSCs were observed using an inverted fluorescence microscope (Leica DFC500, German) and Western blotting was performed to assess the expression of NANOG or PBX1.

2.3. Adipogenesis and Osteogenesis Assays with HF-MSCs. For adipogenic differentiation assays, HF-MSCs ectopically expressing NANOG or overexpressing PBX1, and empty vector (control) were cultured in 24 -well plates (in triplicate) for 2 weeks in adipogenic differentiation medium consisting of High Glucose Dulbecco's Modified Eagle Medium (DMEM; Gibco, Life Technologies, USA) with 10\% FBS (Gibco, USA), $1 \mu \mathrm{M}$ dexamethasone (Sigma-Aldrich, USA), $0.5 \mathrm{mM}$ isobutyl-methylxanthine (Sigma-Aldrich, USA), $10 \mu \mathrm{M}$ insulin (Sigma-Aldrich, USA), and $200 \mu \mathrm{M}$ indomethacin (Sigma-Aldrich, USA). After the cultivation period, cells were stained with Oil Red O (Sigma-Aldrich, USA) to evaluate the formation of lipid droplets and were subsequently imaged using an inverted fluorescence microscope equipped with a digital camera (Leica DFC500, Germany). To quantify adipogenesis, $200 \mu \mathrm{l}$ of isopropanol (Beijing Chemical Factory, China) was added to each of the 24 wells to dissolve the Oil Red $\mathrm{O}$ at room temperature. After $20 \mathrm{~min}$, OD values were measured at a maximum absorption wavelength of $512 \mathrm{~nm}$ using a microplate reader (BioTek, USA), and the concentration of Oil Red O was calculated based on a standard curve that had been created by measuring the optical density of Oil Red O using serial dilutions [31].

For osteogenic differentiation assays, HF-MSCs ectopically expressing NANOG or overexpressing PBX1, and empty vector (control) were cultured in 24-well plates (in triplicate) in osteogenic differentiation medium consisting of High Glucose DMEM (Gibco, Life Technologies, USA) with 10\% FBS (Gibco, USA), $0.1 \mathrm{mM}$ dexamethasone (Sigma-Aldrich, USA), $50 \mathrm{mM}$ ascorbate-2-phosphate (Sigma-Aldrich, USA), and $10 \mathrm{nM} \beta$-glycerophosphate for 4 weeks at $37^{\circ} \mathrm{C}$ with $5 \% \mathrm{CO}_{2}$. At the end of the cultivation period, cells were stained with Alizarin Red S (SigmaAldrich, USA) to assess the formation of mineralized nodules, and cells were imaged using an inverted fluorescence microscope equipped with a digital camera (Leica DFC500, Germany). To quantify osteogenesis, $200 \mu \mathrm{l}$ of $10 \%$ cetylpyridinium chloride (Sigma-Aldrich, USA) was added to each of the 24 wells to dissolve the calcium salt-Alizarin Red S complex at $37^{\circ} \mathrm{C}$ for $30 \mathrm{~min}$. The $\mathrm{OD}$ values of this complex were measured at a maximum absorption wavelength of $558 \mathrm{~nm}$ using a microplate reader, and the concentration of calcium salt-Alizarin Red S complexes was calculated based on a standard curve created by measuring the optical density of a $\mathrm{CaCl}_{2}$-Alizarin Red $\mathrm{S}$ gradient in $10 \%$ cetylpyridinium chloride [31].

2.4. Cell Proliferation and Cell Cycle Assays. To assess cell proliferation, HF-MSCs were seeded at $2 \times 10^{4}$ cells per well in a 24-well plate with culture medium containing DMEM/F12 and 10\% FBS without bFGF and cultured for 5 days. Cells were washed with PBS, digested with $0.25 \%$ trypsin, and counted using a hemocytometer (Qiujing, Shanghai, China) at each time point.

For cell cycle assays, $1 \times 10^{6}$ HF-MSCs transduced with PBX1 (PBX1 group), NANOG (NANOG group), or empty vector (vector group) were harvested and fixed in $70 \%$ ice-cold ethanol at $-20^{\circ} \mathrm{C}$ overnight. The following day, HF-MSCs were washed three times with PBS and incubated in $500 \mu \mathrm{l}$ of propidium iodide with RNase (BD Biosciences, USA) for $15 \mathrm{~min}$ at room temperature in the dark, subsequently subjected to flow cytometry (FACSCalibur; BD Biosciences, USA) and analyzed using CellQuest Pro software (BD Biosciences, USA). The cell proliferation index (PI) was calculated as follows: $\mathrm{PI}=(\mathrm{S}+\mathrm{G} 2 / \mathrm{M}) /(\mathrm{G} 0 /$ $\mathrm{G} 1+\mathrm{S}+\mathrm{G} 2 / \mathrm{M}) \times 100 \%[26]$.

2.5. Senescence-Associated- $\beta$-Galactosidase Assay. The detection of cellular senescence was performed by SA- $\beta$-gal activity assays using a cellular senescence staining kit (Beyotime Biotechnology, China) according to the manufacturer's instructions. After HF-MSCs reached $80 \%$ confluency in a 24-well plate, cells were fixed with the fixative from the kit for $15 \mathrm{~min}$ at room temperature and washed using PBS. Next, cells were cultured in Staining Solution Mix overnight at $37^{\circ} \mathrm{C}$ without $\mathrm{CO}_{2}$. The following day, cells were washed twice with PBS, and SA- $\beta$-gal positive cells were observed and calculated based on three randomly selected bright fields (containing at least 200 cells per field).

2.6. Western Blotting. For Western blotting, $6 \times 10^{5}$ HFMSCs were seeded in a $10 \mathrm{~mm}$ cell culture dish with DMEM/F12 medium supplemented with 10\% FBS and $2 \mathrm{ng} / \mathrm{ml} \mathrm{bFGF}$. When cell confluency reached $80 \%$, cells were digested and washed twice with cold PBS. Then, cells were lysed in $250 \mu \mathrm{l}$ RIPA (Beyotime Biotechnology, China) 
supplemented with $1 \%$ protease inhibitor cocktail (CoWin Biosciences, China) and $1 \%$ phosphatase inhibitor cocktail (CoWin Biosciences, China) on ice for $30 \mathrm{~min}$, and subsequently centrifuged at $15,000 \mathrm{~g}$ for $20 \mathrm{~min}$ at $4^{\circ} \mathrm{C}$. Next, the concentration of the supernatant was examined using an Enhanced BCA Protein Assay Kit (Beyotime Biotechnology, China), and $15 \mu \mathrm{g}$ of protein from each sample was loaded in each well and separated by $10 \%$ SDS polyacrylamide gel electrophoresis. Proteins were then transferred from the gels to polyvinylidene difluoride membranes (Millipore, USA), and membranes were incubated in 5\% nonfat milk powder (Anchor, New Zealand) with tris-buffered saline containing Tween 20 (TBST) at room temperature for $1 \mathrm{~h}$. Next, blots were incubated with primary antibodies in Primary Antibody Dilution Buffer (Beyotime Biotechnology, China) according to the stated antibody dilution ratio. The primary and secondary antibodies used were as follows: AKT (pan) (C67E7) rabbit $\mathrm{mAb}(1: 1000$; CST), phospho-AKT (Ser473) rabbit $\mathrm{mAb}(1: 1000$; CST), NANOG rabbit $\mathrm{mAb}$ ( $1: 1000$; CST), PBX1 rabbit mAb (1:1000, CST), p16 rabbit mAb (1:1000; ProteinTech Group Inc., USA), p21 Waf1/Cip1 (12D1) rabbit mAb (1:1000; CST), p53 mouse mAb (1:1000; Santa Cruz Biotechnology, Inc.), PARP1 rabbit mAb (1:1000; Santa Cruz Biotechnology, Inc.), GAPDH mouse mAb (1:10000; ProteinTech Group Inc.), HRPconjugated AffiniPure Goat Anti-Rabbit IgG $(\mathrm{H}+\mathrm{L})$ (1:5000; ProteinTech Group Inc.), and HRP-conjugated AffiniPure Goat Anti-Mouse IgG (H+L) (1:5000; ProteinTech Group Inc.). Images were obtained using a chemiluminescence imaging analysis system (ECL; Tanon 5200; Shanghai Tianneng Technology Co., Ltd., Shanghai, China), and band intensity was analyzed with Tanon Gis analytical software (Shanghai Tianneng Technology Co., Ltd., Shanghai, China).

2.7. Dual-Luciferase Reporter Gene Assays. NANOG ectopic expression plasmid- and vector-expressing HF-MSCs were cultured in a 24-well plate and transfected with plasmids that included the $P B X 1$ promoter pGL3-Basic vector $(0.9 \mu \mathrm{g} /$ well $)$ and pRL-TK vector $(0.1 \mu \mathrm{g} /$ well; Youbio Biological Technology Co., Ltd.). The empty pGL3-Basic vector $(0.9 \mu \mathrm{g} /$ well $)$ and $\mathrm{pRL}$-TK vector $(0.1 \mu \mathrm{g} /$ well $)$ were used as the control conditions. Transfection reagent $(1.1 \mu \mathrm{l})$ was added to each well (GeneCopoeia, China). After $6 \mathrm{~h}$, the medium was replaced with fresh DMEM/F12 and 10\% FBS. The cells were lysed and the supernatant was then harvested, and relative light units (RLUs) were measured after $36 \mathrm{~h}$ using the Cytation 3 Cell Imaging Multi-Mode Reader (BioTek Instruments, USA) according to the specifications of the Dual Luciferase Reporter Gene Assay Kit (Beyotime Biotechnology, China).

2.8. Inhibition of PI3K/AKT Signaling with an AKT Inhibitor or shRNA. The inhibitor LY294002 (MedChemExpress, USA) was used to block the PI3K/AKT signaling pathway in order to inhibit the phosphorylation of AKT. The optimal treatment concentration and time for LY294002 were determined by Western blotting (data not shown). HF-MSCs that were ectopically expressing NANOG were treated with $P B X 1$ shRNA (AGCTGTCACTGCTACCAATGT) or AKT shRNA
(ATCGCTTCTTTGCCGGTAT) to inhibit the expression of PBX1 or AKT, respectively (GeneChem, Shanghai, China). In addition, the appropriate shRNA control was also used. Transfection and verification were performed as previously mentioned in the section of lentiviral plasmid construction and transduction.

2.9. Statistical Analyses. Data were statistically analyzed using SPSS version 21.0 (IBM Corp., Armonk, NY, USA). The data are presented as the mean \pm standard deviation (SD). Differences between two groups were compared using the independent samples' $t$-test, whereas differences between multiple groups were compared by one-way analysis of variance (one-way ANOVA). $P<0.05$ was considered to be statistically significant. All results were repeated independently a minimum of three times, except where indicated.

\section{Results}

3.1. Isolation and Identification of HF-MSCs. HF-MSCs migrated from human hair follicles 10-14 days after seeding in a 24-well plate and displayed a fibroblastlike morphology (Figures 1(a) and 1(b)). Immunofluorescence and flow cytometry assays showed that HF-MSCs expressed CD44, CD73, CD90, and CD105 with low expression of CD31 (Figures 1(c) and 1(d)). Moreover, Oil Red $\mathrm{O}$ and Alizarin Red S staining showed that HFMSCs exhibited osteogenic and adipogenic differentiation potential, respectively (Figures 1(e) and 1(f)).

3.2. Ectopic NANOG Expression or PBX1 Overexpression Promotes HF-MSCs Proliferation and Cell Cycle Transition from G0/G1 to $S$ Phase. Ectopic NANOG expression or PBX1 overexpression via lentivirus transduction was visualized by immunofluorescence imaging and measured by Western blotting (Figures 2(a) and 2(b)). In addition, the results of Western blotting indicated that there was no expression of NANOG and low expression of PBX1 in HF-MSCs (Figure 2(b)).

Cell growth assays showed that the ectopic expression of NANOG or the overexpression of PBX1 significantly increased the quantity of HF-MSCs by 1.4- and 1.2-fold, respectively, in comparison to cells in the vector group (control) on day 8 (Figure 2(c)). Cell cycle assays also showed that the ectopic expression of NANOG or the overexpression of PBX1 also significantly increased the proportion of HFMSCs in the S phase to $14.56 \pm 2.43 \%$ in the NANOG group and $11.79 \pm 1.84 \%$ in the PBX1 group in comparison to that of the vector group $(7.87 \pm 1.72 \%)$. Thus, ectopic NANOG expression or PBX1 overexpression significantly increased the proliferation index by 1.43 - and 1.29-fold, respectively, in comparison to that of the vector group $(P<0.01$; Figures 2(d) and 2(e)).

3.3. Ectopic NANOG Expression or PBX1 Overexpression Promotes Osteogenesis, Inhibits Adipogenesis, and Delays Cellular Senescence. Oil Red O and Alizarin Red S staining showed that ectopic NANOG expression or PBX1 overexpression decreased lipid droplet accumulation by $0.71 \pm$ 0.14 - and $0.62 \pm 0.13$-fold, respectively, in comparison to 


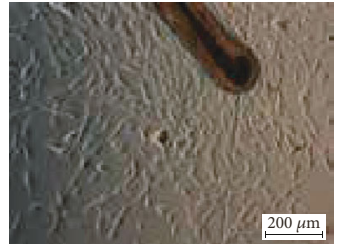

(a)

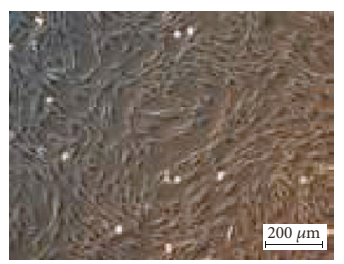

(b)
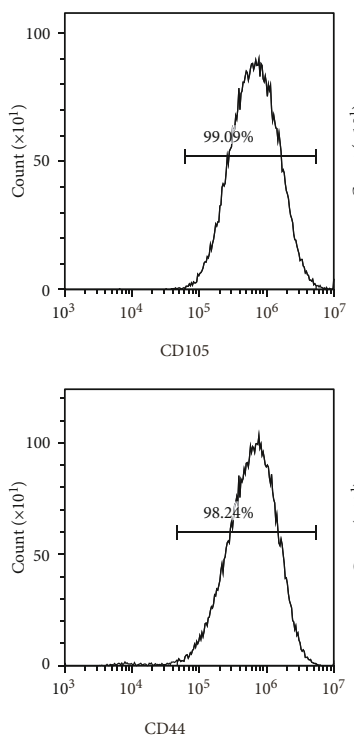

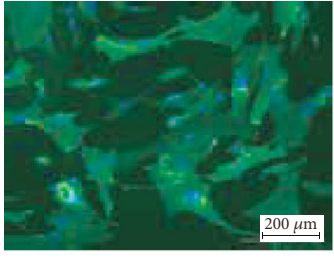

CD105

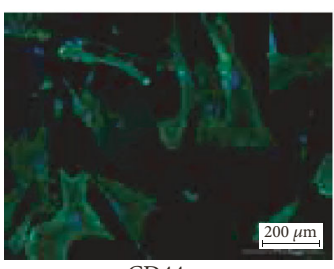

CD44

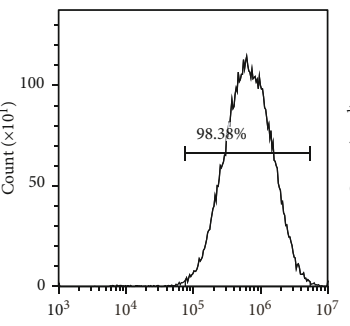

CD90

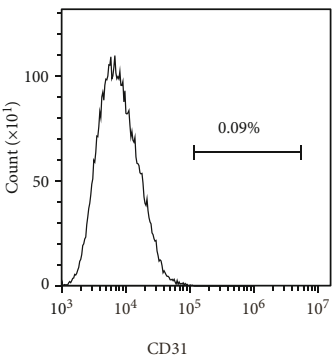

(d)

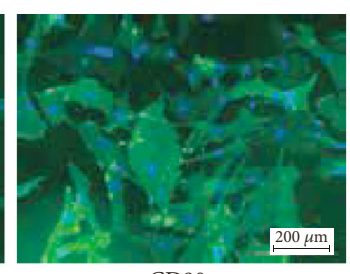

CD90

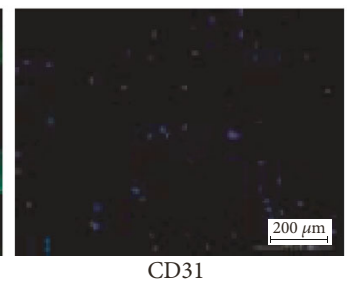

(c)

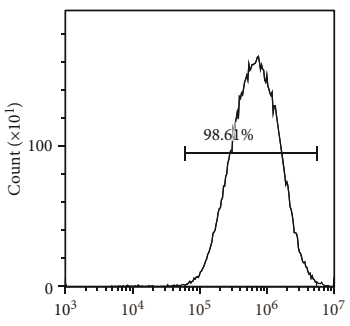

CD73

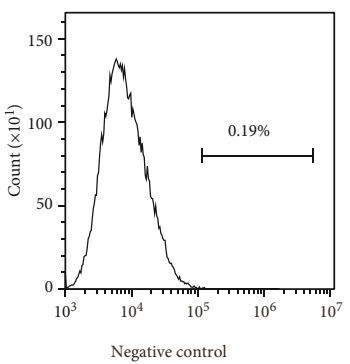

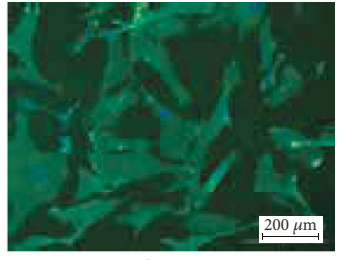

$\mathrm{CD} 73$

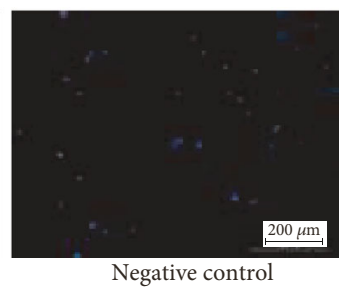

Negative control

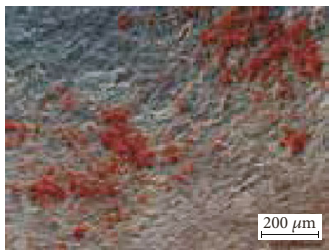

(e)

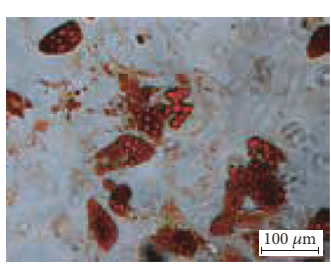

(f)

Figure 1: Isolation and identification of hair follicle-derived mesenchymal stem cells (HF-MSCs). (a, b) Cells that migrated from hair follicles exhibited fibroblast-like characteristics. Scale bar $=200 \mu \mathrm{m}$. (c) Immunofluorescence assay showing that the cells expressed CD44, CD73, CD90, and CD105 but not CD31. (d) Flow cytometry was used to measure the cell surface expression of MSC markers. (e) HF-MSCs were cultured in osteogenic differentiation media and stained (red) with Alizarin Red S. Scale bar $=200 \mu \mathrm{m}$. (f) HF-MSCs were cultured in adipogenic differentiation media and stained (red) with Oil Red O. Scale bar $=100 \mu \mathrm{m}$.

that of the vector group ( $P<0.05$ and $P<0.01$, respectively) and also increased calcium nodal formation in HF-MSCs by $2.18 \pm 0.05$ - and $1.78 \pm 0.18$-fold $(P<0.001$ and $P<0.01)$, respectively (Figures 3(a)-3(d)). Furthermore, ectopic NANOG expression or PBX1 overexpression significantly decreased the percentage of SA- $\beta$-gal-positive cells to 28.33 $\pm 1.73 \%$ at passage 6 and $81.33 \pm 3.46 \%$ at passage 14 (PBX1 vs vector, $P<0.01$ ) or $30.33 \pm 1.73 \%$ at passage 6 and $80.33 \pm 2.31 \%$ at passage 14 (NANOG vs vector, $P<0.01$ ) in comparison to $44.67 \pm 2.02 \%$ at passage 6 and $98.33 \pm 0.67 \%$ at passage 14 in the vector group (Figures 3(e) and 3(f)).

3.4. Subculture Decreases $P B X 1$ and Increases the Expression of p16, p53, and p21 in HF-MSCs. Western blotting was used to elucidate the relationship between PBX1, cell passage, and proteins related to cell senescence. Our results show that with passage, the relative expression of PBX1 in HF-MSCs was significantly decreased at passage 7 by $0.6 \pm 0.13$-fold $(P<0.01)$ and at passage 10 by $0.25 \pm 0.07$-fold $(P<0.001)$ in comparison to that of passage 4 . In contrast, the expression of p16, p53, and p21 was significantly increased with passage in HF-MSCs by $1.34 \pm 0.01$-fold at passage $7(P<0.001)$ and $1.49 \pm 0.09$-fold at passage $10(P<0.001)$ for $\mathrm{p} 16,1.54 \pm 0.03$ -fold at passage $7(P<0.001)$ and $2.07 \pm 0.04$-fold at passage $10(P<0.001)$ for $\mathrm{p} 53$, and $1.34 \pm 0.35$-fold at passage 7 $(P=0.10)$ and $2.08 \pm 0.54$-fold at passage $10(P<0.01)$ for $\mathrm{p} 21$, in comparison to the expression at passage 4 (Figure 3(g)).

\subsection{Ectopic NANOG Expression or PBX1 Overexpression} Upregulates PARP1 and $p$-AKT and Downregulates p16, $p 53$, and $p 21$. As AKT signaling is reportedly involved in cell 

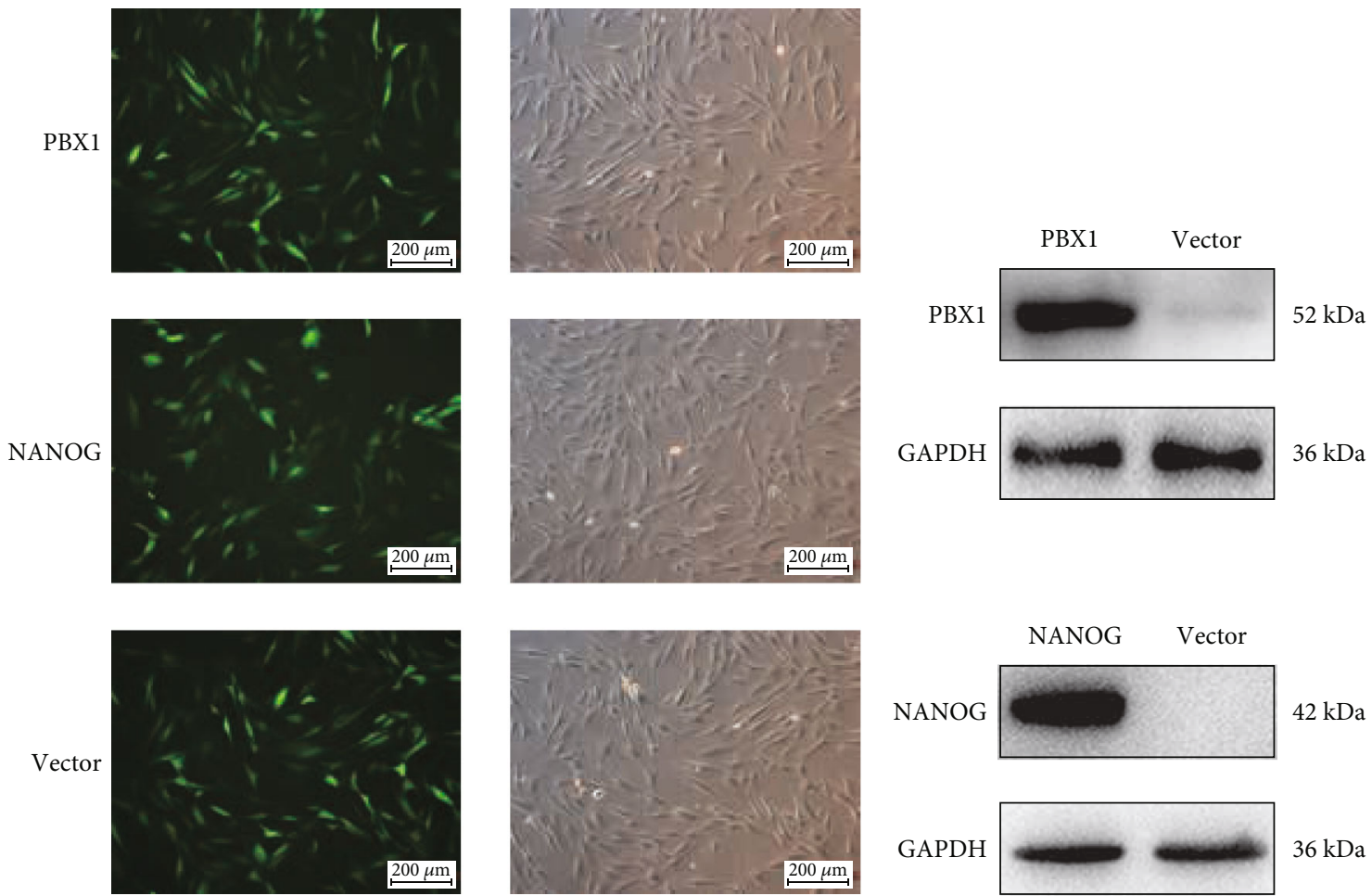

(a)

(b)

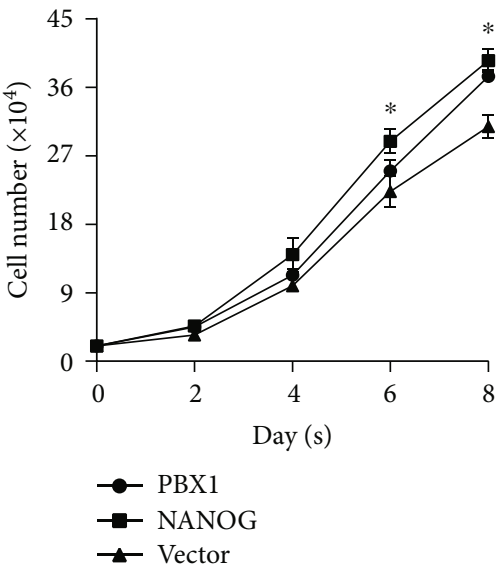

(c)

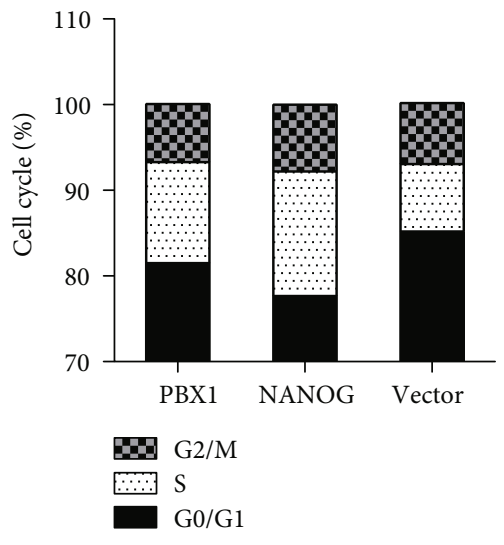

(d)

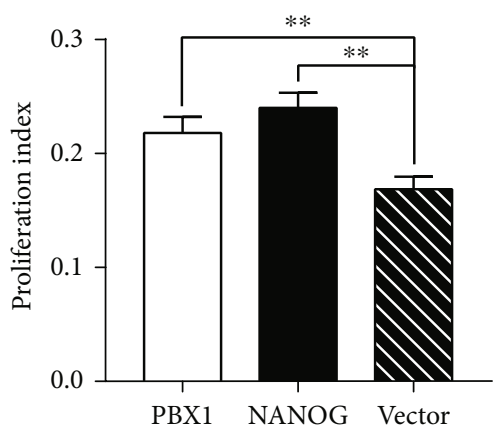

(e)

FIGURE 2: Ectopic NANOG expression and PBX1 overexpression promote proliferation and regulate the cell cycle of hair follicle-derived mesenchymal stem cells (HF-MSCs). (a, b) Images and Western blotting showed ectopic NANOG expression or PBX1 overexpression after lentiviral transduction. Scale bar $=200 \mu \mathrm{m}$. (c) Growth curves showed that NANOG and PBX1 promoted proliferation in comparison to that of control (vector only) cells. (d-e) Cell cycle was measured by flow cytometry, which revealed an effect on cell cycle phase distribution. The proliferation indexes of NANOG- and PBX1-expressing cells were higher than that of the control (vector; ${ }^{*} P<0.05$, $\left.{ }^{* *} P<0.01\right)$.

senescence, we aimed to determine if NANOG or PBX1 delays HF-MSC senescence through the activation of this pathway. Indeed, Western blotting showed that NANOG and PBX1 significantly increased PARP1 expression by $1.64 \pm 0.09$-fold $(P<0.01)$ and $1.75 \pm 0.14$-fold $(P<0.01)$, respectively, and the expression of $\mathrm{p}-\mathrm{AKT} / \mathrm{AKT}$ by $3.14 \pm$ 0.65 -fold $(P<0.05)$ and $3.35 \pm 0.46$-fold $(P<0.01)$, respectively, in comparison to the empty vector. However, NANOG and PBX1 decreased p16 expression by $0.38 \pm 0.18$-fold
$(P<0.01)$ and $0.49 \pm 0.26$-fold $(P<0.05)$, respectively, $\mathrm{p} 21$ expression by $0.57 \pm 0.24$-fold $(P<0.05)$ and $0.58 \pm 0.24$ -fold $(P<0.05)$, respectively, and p53 expression by $0.60 \pm$ 0.16 -fold $(P<0.05)$ and $0.58 \pm 0.25$-fold $(P<0.05)$, respectively (Figures 4(a) and 4(b)).

3.6. Blocking p-AKT or AKT Knockdown Significantly Downregulates PBX1, Upregulates p16 and p21, and Enhances Cell Senescence. To explore the relationship 


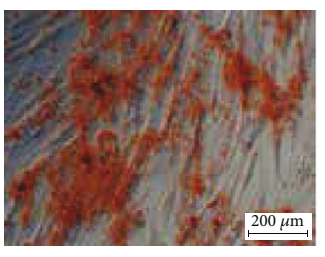

PBX1

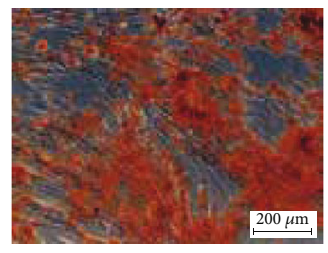

NANOG

(a)

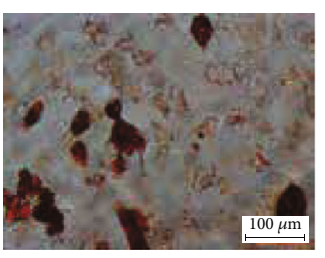

PBX1

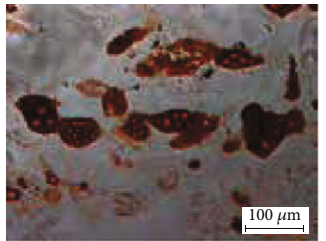

NANOG (c)

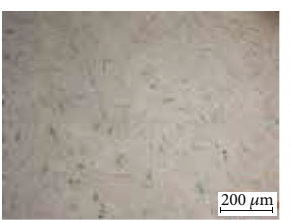

P6 PBX1

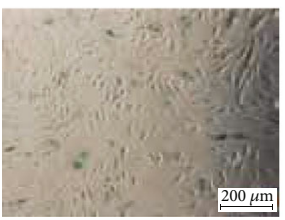

P14 PBX1

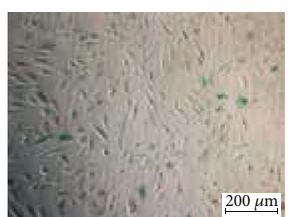

P6 NANOG

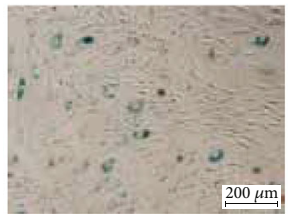

P14 NANOG

(e)

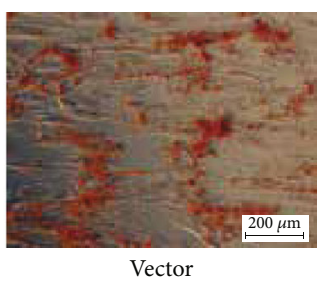

Vector

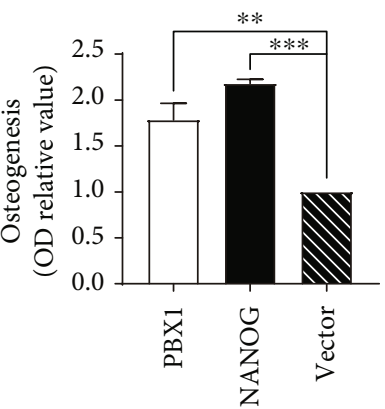

(b)

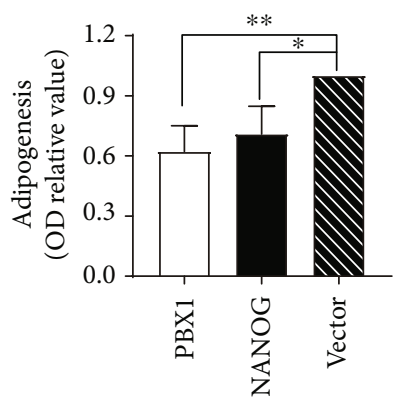

(d)

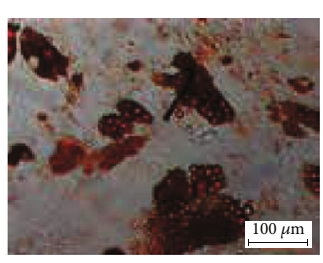

Vector

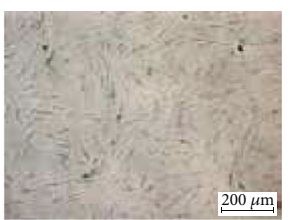

P6 vector

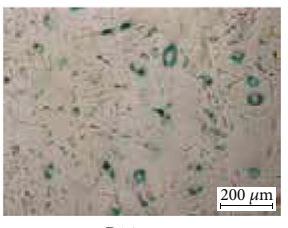

P14 vector
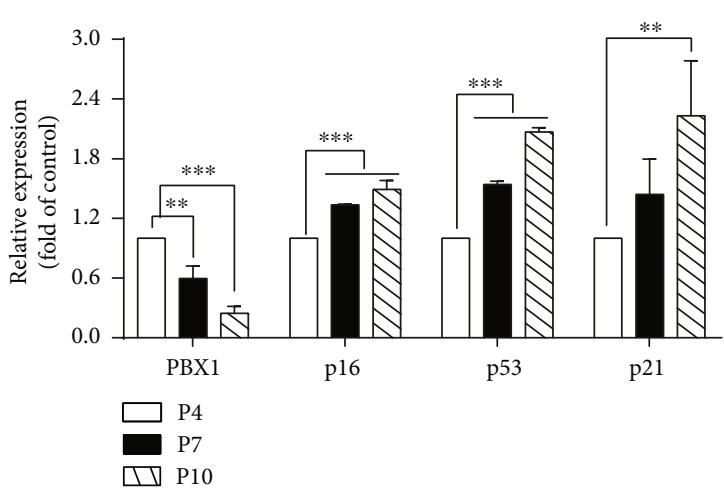

(g)
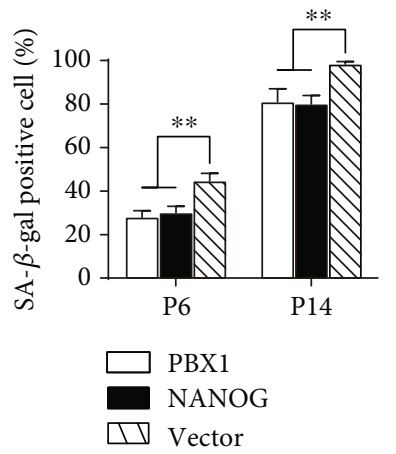

(f)

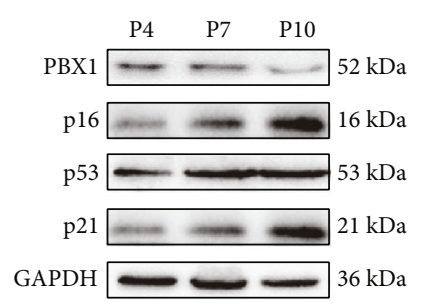

FIGURE 3: Ectopic NANOG expression and PBX1 overexpression regulate osteogenesis and adipogenesis, respectively. (a, b) NANOG and PBX1 promoted osteogenesis based on Alizarin Red S staining and quantitative detection. Scale bar $=200 \mu \mathrm{m}$. (c, d) NANOG and PBX1 repressed adipogenesis based on Oil Red $\mathrm{O}$ staining and quantitative detection. Scale bar $=100 \mu \mathrm{m}$. (e, f) Blue cells were considered to be positive for senescence based on senescence-associated $\beta$-galactosidase (SA- $\beta$-gal) staining. There were fewer positive cells in NANOG- and PBX1-expressing groups in comparison to the vector control group at both passages 6 and 14 . Scale bar $=200 \mu \mathrm{m}$. (g) Western blotting showed that PBX1 expression decreased and senescence-related protein expression increased with increasing passages $\left({ }^{*} P<0.05,{ }^{* *} P<0.01,{ }^{* * *} P<0.001\right)$. 


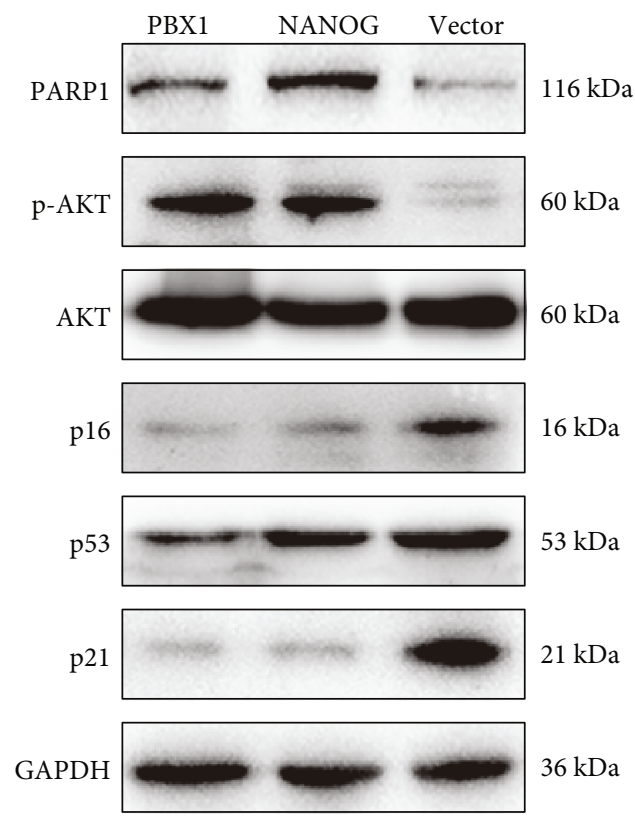

(a)
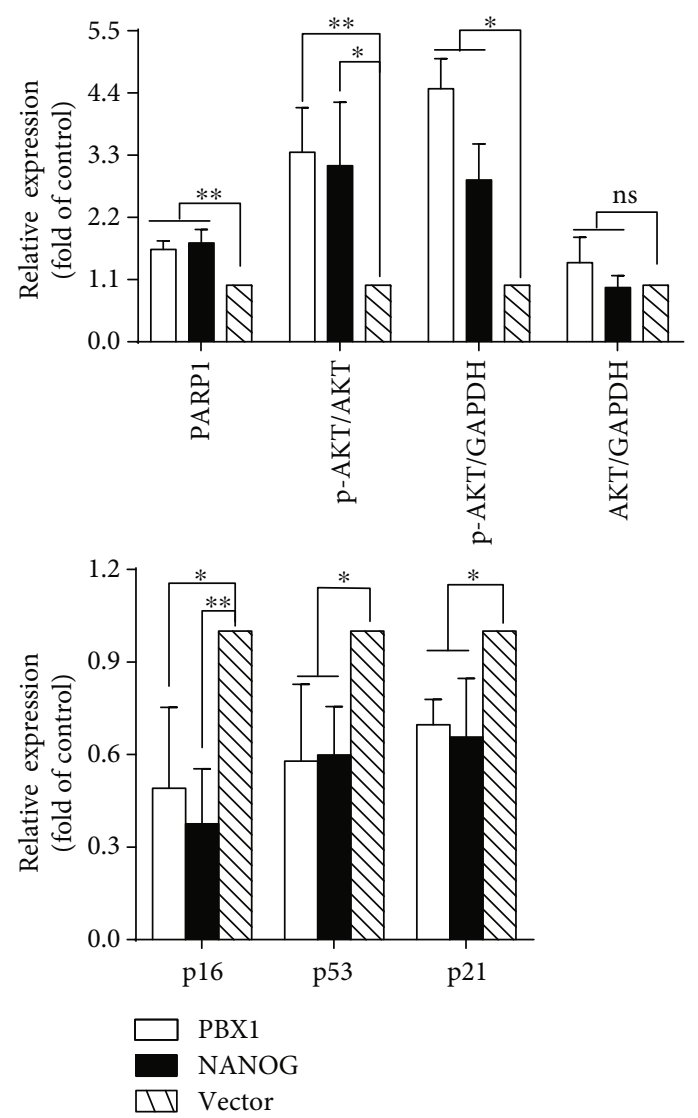

(b)

FIGURE 4: Ectopic NANOG expression and PBX1 overexpression delayed the senescence of hair follicle-derived mesenchymal stem cells (HFMSCs) via the AKT pathway. ( $a, b)$ Western blotting showed that NANOG and PBX1 expression upregulated PARP1 and AKT signaling pathways and downregulated the expression of p16, p53, and p21 in comparison to cells in the vector control group $\left({ }^{*} P<0.05,{ }^{* *} P<0.01\right)$.

between PBX1 and p-AKT, p-AKT was blocked using the PI3K/AKT inhibitor LY294002. Western blotting showed that treating HF-MSCs that were ectopically expressing NANOG with this compound significantly reduced PBX1 expression to $69.38 \pm 6.62 \%$ of control levels $(P<0.01)$ but increased the expression of p16, p53, and p21 to $162.91 \pm$ $19.72 \%(P<0.05), 123.43 \pm 6.25 \%(P<0.05)$, and $160.99 \pm$ 9.29\% $(P<0.01)$ of control levels, respectively. Furthermore, blocking p-AKT significantly increased SA- $\beta$-gal-positive cell numbers from $43.33 \pm 2.40 \%$ to $82.67 \pm 1.20 \%$ $(P<0.05$; Figures 5(a) $-5(\mathrm{c}))$. To confirm these results, total AKT was knocked down by RNA interference in HF-MSCs that were ectopically expressing NANOG. Our results showed that the inhibition of AKT significantly reduced PBX1, p-AKT, and total AKT expression to $29.55 \pm 5.26 \%$ $(P<0.001), 59.16 \pm 1.86 \%(P<0.001)$, and $64.36 \pm 4.47 \%$ $(P<0.001)$ of control levels, respectively, but increased the expression of p16 and p21 to $149.17 \pm 14.76 \%(P<0.05)$ and $143.58 \pm 7.84 \%(P<0.01)$ of control levels, respectively. As expected, total AKT knockdown led to a significant increase in SA- $\beta$-gal-positive cells from $47.67 \pm 1.45 \%$ to $87.33 \pm 1.45 \%(P<0.001$; Figures $5(\mathrm{~d})-5(\mathrm{f}))$.

3.7. Ectopic NANOG Expression Significantly Upregulates PBX1 and Increases PBX1 Promoter Activity. To elucidate the relationship between NANOG and PBX1, Western blotting and dual luciferase reporter assays were performed. Western blotting showed that ectopic NANOG expression significantly upregulated PBX1 expression by $4.11 \pm 0.54$-fold in comparison to that of the control group $(P<0.001$; Figures 6(a) and 6(b)). Dual-luciferase reporter gene assays showed that ectopic NANOG expression significantly increased $P B X 1$ promoter activity by $1.99 \pm$ 0.43 -fold in comparison to that of the control group $(P<0.05)$, suggesting that NANOG may increase $P B X 1$ expression (Figure 6(c)).

3.8. Knockdown of PBX1 Downregulates p-AKT, Upregulates p16 and p21, and Enhances Cell Senescence. To further elucidate the relationship between NANOG and PBX1, HF-MSCs that were ectopically expressing NANOG were transduced with $P B X 1$ shRNA. Western blotting showed that the inhibition of $P B X 1$ by RNA interference significantly decreased the expression of p-AKT to $52.47 \pm 11.13 \%$ of control levels $(P<0.01)$ and increased the expression of p16 and $\mathrm{p} 21$ to $138.30 \pm 13.43 \%(P<0.05)$ and $201.21 \pm 26.56 \%(P<0.01)$ of control levels, respectively (Figures 6(d) and 6(e)). Consistently, $P B X 1$ knockdown significantly increased the quantity of SA- $\beta$-gal-positive cells from $64.33 \pm 2.33 \%$ to $83.67 \pm$ 2.73\% $(P<0.01$; Figure 6(f)). 


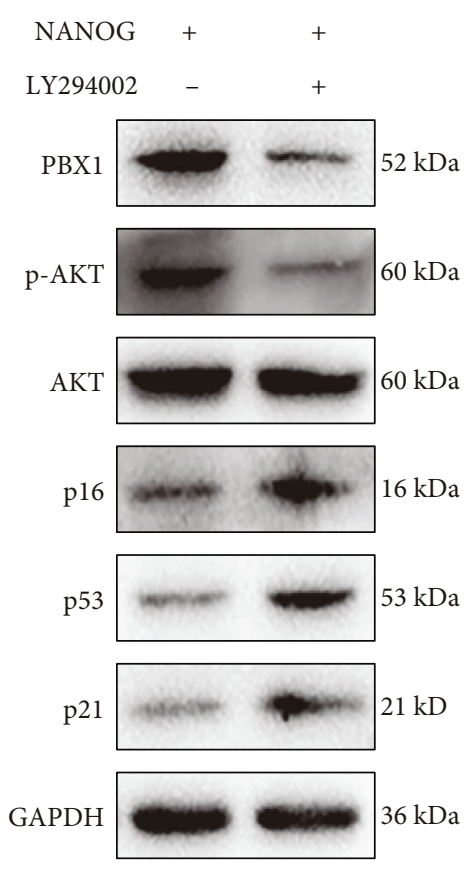

(a)

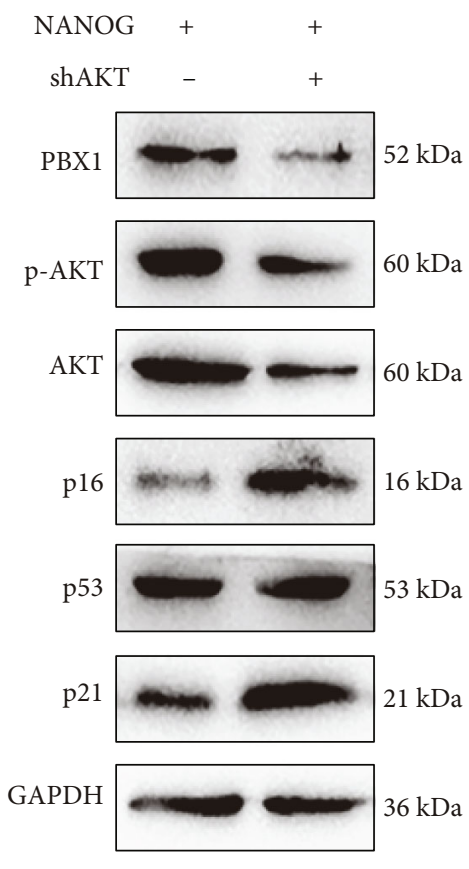

(d)
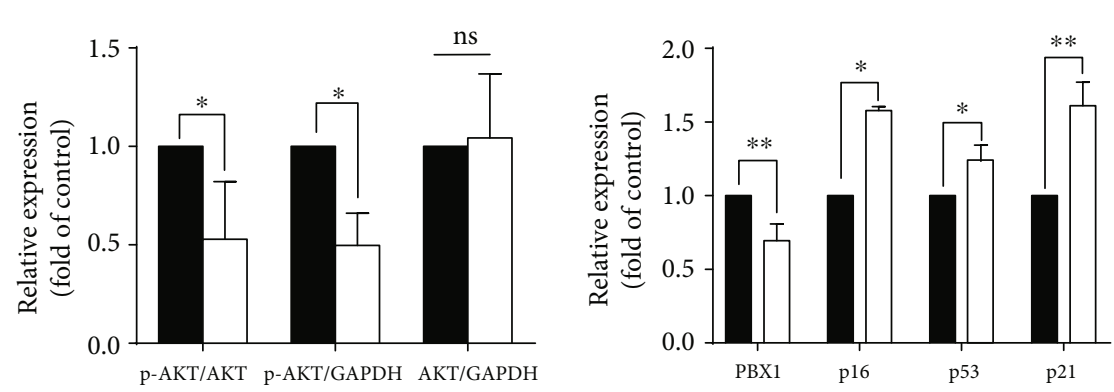

NANOG-LY294002

NANOG

(b)

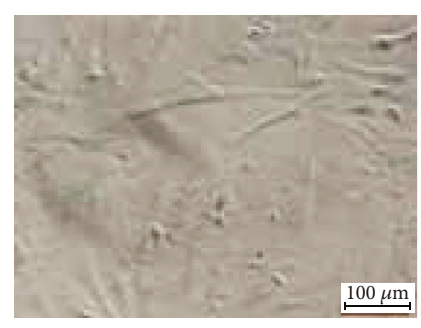

NANOG

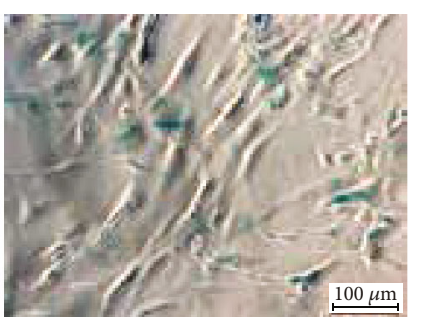

NANOG-LY294002

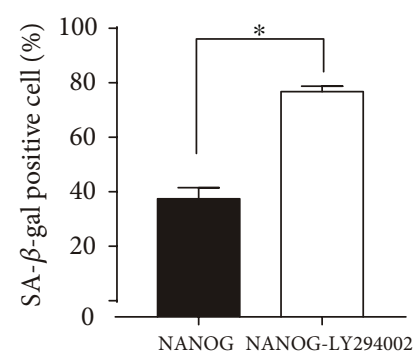

(c)

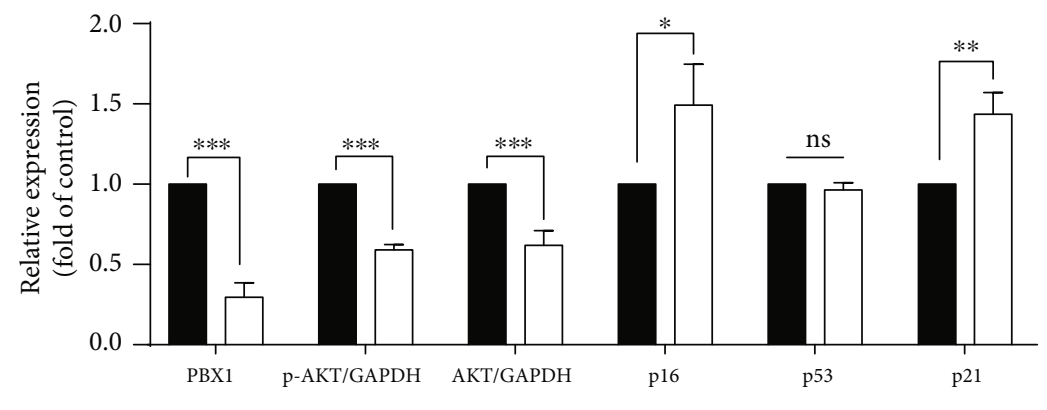

NANOG-ShAKT

NANOG-shNC

(e)

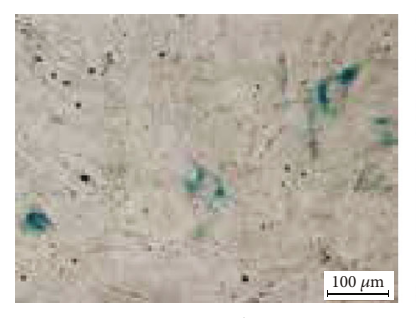

NANOG-shNC

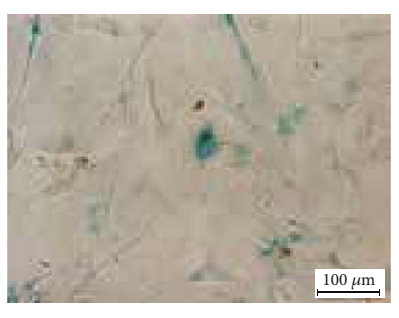

NANOG-shAKT

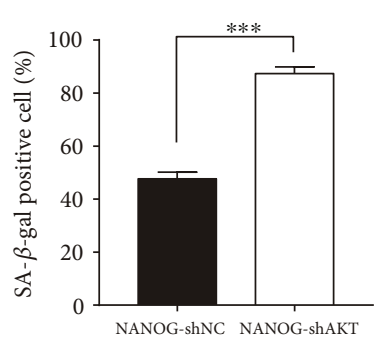

(f)

FIGURE 5: Blocking p-AKT or AKT knockdown significantly downregulates PBX1; upregulates p16, p53, and p21; and enhances cell senescence. ( $\mathrm{a}, \mathrm{b})$ HF-MSCs that were ectopically expressing NANOG were treated with the PI3K/AKT inhibitor LY294002 (40 $\mu \mathrm{M}, 24 \mathrm{~h}$ ). Western blotting shows that after the inhibition of PI3K/AKT signaling, the expression of p16, p53, p21, PBX1, and p-AKT was significantly altered. (c) Senescence-associated $\beta$-galactosidase (SA- $\beta$-gal) staining was used to examine cellular senescence after inhibiting the PI3K/AKT pathway. Scale bar $=100 \mu \mathrm{m}$. (d, e) HF-MSCs that were ectopically expressing NANOG were treated with AKT shRNA. Western blotting shows that knocking down AKT resulted in the downregulation of PBX1 and an increase in the expression of p16 and p21. Differences in the expression of p16, p21, PBX1, AKT, and p-AKT were statistically significant. (f) SA- $\beta$-gal staining was used to examine cellular senescence after knocking down $A K T$. Scale bar $=100 \mu \mathrm{m} .\left({ }^{*} P<0.05,{ }^{* *} P<0.01,{ }^{* * *} P<0.001\right)$. 


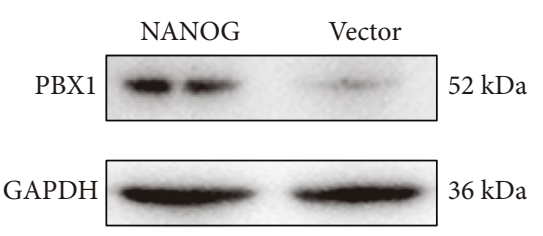

(a)

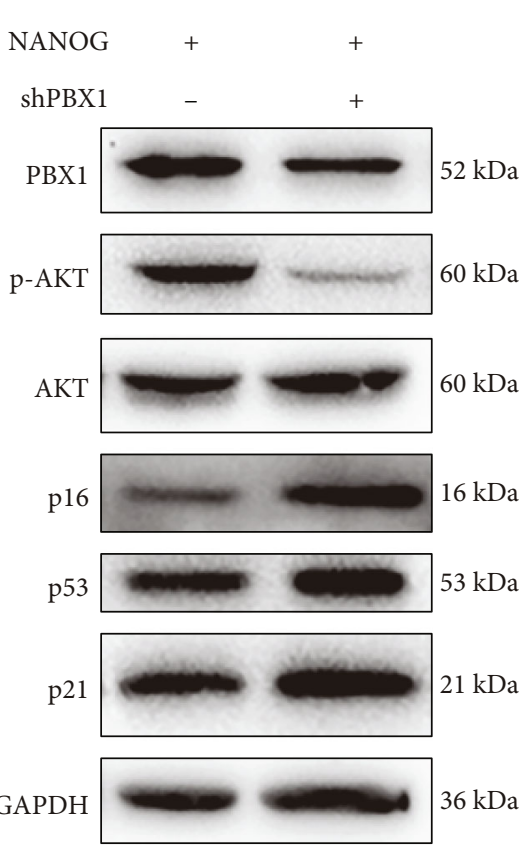

(d)

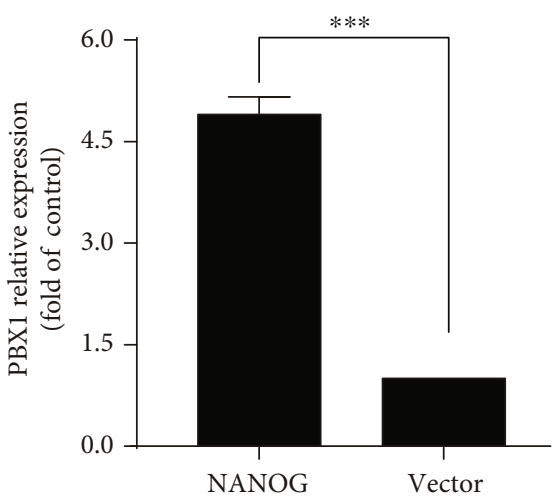

(b)

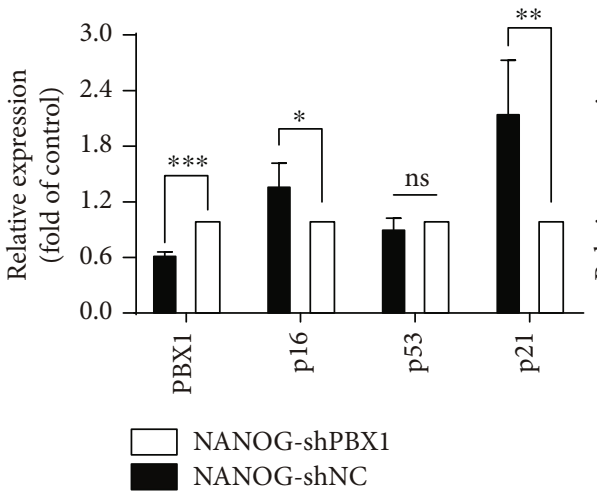

(e)

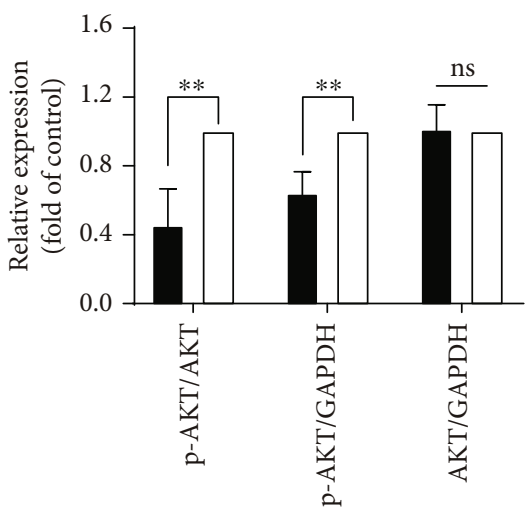

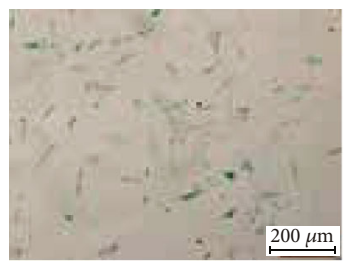

NANOG-shPBX1

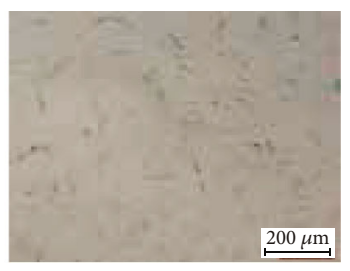

NANOG-shNC

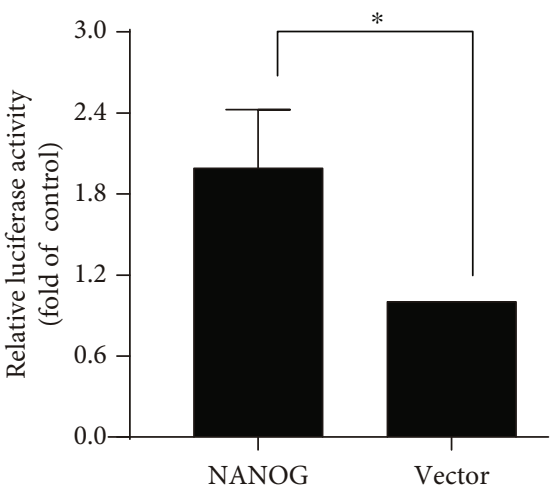

(c)

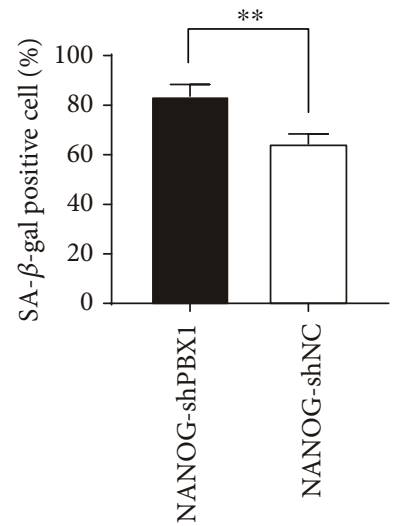

(f)

FIGURE 6: NANOG upregulates the expression of PBX1, while ectopic NANOG expression with $P B X 1$ shRNA downregulates the phosphorylation of AKT. (a, b) Western blotting shows that ectopic NANOG expression upregulates PBX1 $\left({ }^{* * *} P<0.001\right)$. (c) After $36 \mathrm{~h}$ of plasmid transfection ( $P B X 1$ promoter pGL3-Basic vector and pRL-TK vector), dual luciferase assays were performed, and luciferase activities (relative light units) were measured in comparison to those of the vector control group $\left({ }^{*} P<0.05\right)$. (d) Hair follicle-derived mesenchymal stem cells (HF-MSCs) ectopically expressing NANOG were treated with PBX1 shRNA. Western blotting shows that PBX1 shRNA downregulated p-AKT and increased the expression of p16 and p21. (e) Differences in the expression of p16, p21, and p-AKT, but not p53, were statistically significant. (f) Senescence-associated $\beta$-galactosidase (SA- $\beta$-gal) staining was used to examine cellular senescence. Scale bar $=200 \mu \mathrm{m} .\left({ }^{*} P<0.05,{ }^{* *} P<0.01,{ }^{* * *} P<0.001\right)$.

\section{Discussion}

The hair follicle is an appendage of human skin and contains abundant MSCs. HF-MSCs play crucial roles in the generation of hair follicles and hair cycle progression. HF-MSCs can be used as cell sources for the engineering of vascular tis- sue and angiogenesis, neural regeneration, and hair follicle reconstruction, and the delivery and controlled release of insulin, resulting in tremendous therapeutic potential in clinical settings [32-35].

As a homeodomain transcription factor, NANOG is crucial for maintaining the self-renewal and pluripotency of 
ESCs. Our results demonstrated that there was no endogenous expression of NANOG in HF-MSCs in agreement with Shahini et al.'s study [10]. Moreover, there was no endogenous expression of NANOG in BM-MSCs [12]. Not all multipotential stem cells express NANOG based on the above research. Therefore, we did not downregulate the expression of NANOG.

Han et al. [12] suggested that NANOG might prevent senescence in BM-MSCs and improve proliferation and the capacity for myogenic differentiation. In agreement with a study by the Andreadis group, which suggested that ectopic NANOG expression is able to reverse organismal aging in BM-MSCs, our study showed that the ectopic expression of NANOG or the overexpression of PBX1 results in enhanced AKT phosphorylation, increased HF-MSC proliferation, and decreased cellular senescence at early and late passages, followed by a decrease in markers associated with cellular senescence [36], such as p16, p53, and p21. It has been reported that $\mathrm{p} 16^{\mathrm{INK} 4 \mathrm{a}}$ - a gene related to antiaging-acts as a cyclin-dependent kinase inhibitor and is crucial for cellcycle progression and cellular senescence [37]. Although both $\mathrm{p} 27^{\mathrm{KIP} 1}$ and $\mathrm{p} 21^{\mathrm{CIP} / \mathrm{WAF}}$ are involved in regulating the G1 to $S$ transition, we did not find that NANOG represses cellular senescence by downregulating $\mathrm{p} 27^{\mathrm{KIP} 1}$ in $\mathrm{HF}$ MSCs. It has been suggested that this process is likely celltype specific, which could result in the induction of various patterns of $\mathrm{p} 27^{\mathrm{KIP} 1}$ expression. In addition, it has been shown that p21 is downstream of p53 during the regulation of the G1 phase of cell cycle [13]. Furthermore, our results show that NANOG and PBX1 upregulate poly (ADP-ribose) polymerase1 (PARP1) - a key enzyme involved in DNA repair [38] - and attenuate HF-MSC senescence. In agreement with our results, it has been shown that PARP1 activity decreases with age [39] and that a deficiency of PARP1 accelerates aging and reduces the life span of mice [40].

In this study, we found that ectopic NANOG expression or PBX1 overexpression could significantly enhance osteogenesis and decrease adipogenesis, in agreement with previously published studies that show that PBX1 represses adipocyte differentiation by directly regulating PPAR $\gamma$ after the embryonic stage [19], and that NANOG enhances osteogenesis by regulating the BMP pathway [41]. Humans are prone to weight gain and metabolic bone diseases such as osteoporosis as they age $[42,43]$, and the differentiation potential of BM-SMCs towards an osteogenic lineage decreases or is lost with age [44]. It has also been reported that Sirt3 is involved in age-related adipogenesis and osteoclastogenesis in aging male mice [43]. The same phenomenon of impaired osteogenic and activated adipogenic processes can be observed in aged animals [45]. These data suggest a negative association between adipogenesis and osteogenesis that occurs with aging.

Our results indicate that NANOG and PBX1 are key transcription factors involved in the antiaging process. Moreover, we found that PBX1 expression in HF-MSCs was remarkably decreased with cell subculture, suggesting that it plays a role in maintaining these cells in a proliferative and multipotent state. Most notably, we found that the ectopic expression of NANOG significantly upregulates PBX1 and results in a decrease in the expression of p16 and p21. The upregulation of $P B X 1$ was further confirmed by dual luciferase assays, as NANOG overexpression significantly increased $P B X 1$ promoter activity, indicating that it might be upstream of $P B X 1$. We performed RNA interference assays to confirm these findings and observed that, as expected, $P B X 1$ knockdown in HF-MSCs that were ectopically expressing NANOG significantly reduced the expression of $\mathrm{p}-\mathrm{AKT}$, increased the expression of p16 and p21, and promoted cellular senescence. These results suggest that NANOG attenuates cellular senescence via the PBX1-mediated regulation of AKT signaling and that PBX1 plays a vital role in attenuating HF-MSC senescence. Indeed, further investigation confirmed that the expression of PBX1 was remarkably decreased over time when subculturing HF-MSCs. A study by Li and Wang [20] showed that both NANOG and PBX1 play crucial roles as transcription factors in maintaining ESCs in a pluripotent state and that there is a positive interaction feedback loop between these makers based on a global sensitivity analysis. However, few studies have reported on the relationship between NANOG and PBX1 in MSCs. Both Boyer et al. [22] and Piestun et al. [23] found that the $P B X 1$ promoter is a target of NANOG in NIH3T3 and human ESCs based on a genome-scale location analysis. Moreover, both KLF4 and PBX1 have been shown to collaboratively bind the NANOG promoter to subsequently regulate gene expression in human ESCs [21]. This was our impetus for exploring the relationship between NANOG and PBX1.

Surprisingly, we found LY294002 increased p53 as well as p16 and p21; AKT knockdown increased only p16 and p21 but p53. These results are consistent with the report of Kim et al.'s group, showing that AKT directly phosphorylates $\mathrm{p} 21^{\mathrm{WAF1}}$ but $\mathrm{p} 53$ [46]. This is probably because AKT is an upstream of $\mathrm{p} 21^{\mathrm{WAF} 1}$ [47] and LY294002 is a specific inhibitor of PI3K not AKT. Another reason would be that AKT knockdown results in little AKT expression in cellular cytoplasm, then repeated phosphorylation and dephosphorylation by PIP3 or PHLPP1 probably affecting the stability and function of the modified AKT, therefore decreasing the inhibition of p53 [48]. The results of our study also showed that when PBX1 was knocked down after NANOG ectopic expression, the expression of p16 and p21 increased, and p53 expression showed no statistical difference. Accordingly, NANOG could affect AKT phosphorylation, p16 and p21 expression through $\mathrm{PBX} 1$, while NANOG did not regulate p53 expression through PBX1. The reason behind this phenomenon is that PBX1 knockdown reduced $\mathrm{p}$-AKT expression, which leads to a similar consequence as AKT knockdown. The result is consistent with our group's research which showed that PBX1 enhances HF-MSCs proliferation by activating $\mathrm{AKT}$ and downregulation of p16 and p21 [49]. These results also suggested that there was a feedback loop between PBX1 and AKT signaling.

Although stem cell therapy holds promise for regenerative medicine, stem cells that are derived from aged donors or harvested by repeated subculture tend to lose their capacity for proliferation and multipotent differentiation, which not only compromises their therapeutic efficacy but also raises safety concerns for stem cell therapy. This necessitates 
the development of strategies for maintaining stem cells in a multipotent and proliferative state. Collectively, our study confirms the presence of a NANOG/PBX1/AKT pathway and a feedback interaction loop between PBX1 and AKT in HF-MSCs. The NANOG/PBX1/AKT pathway plays an important role in attenuating HF-MSC senescence, while the feedback interaction loop between PBX1 and AKT is mutually beneficial for maintaining HF-MSCs in a highly proliferative state with differentiation potential. We believe that our study makes an important contribution to antiaging research and regeneration medicine because there are few previous studies on the role and molecular mechanisms of NANOG in delaying senescence of HF-MSCs.

\section{Conclusion}

This study is the first to report the existence of a NANOG/PBX1/AKT pathway and a feedback interaction loop between PBX1 and AKT in HF-MSCs. The NANOG/PBX1/AKT pathway plays an important role in attenuating HF-MSC senescence, while the feedback loop between PBX1 and AKT is mutually beneficial for maintaining HF-MSCs in a highly proliferative state with the multipotential capacity.

\section{Data Availability}

The data used to support the findings of this study are available from the corresponding author upon request.

\section{Conflicts of Interest}

The authors declare no conflict of competing and financial interest.

\section{Authors' Contributions}

Feilin Liu and Jiahong Shi contributed equally to this work. Feilin Liu and Jiahong Shi are co-first authors. Guanfang Su and Jinyu Liu are cocorresponding authors.

\section{Acknowledgments}

This work was supported by the China National Natural Science Foundation (81573067), the Joint Construction Project between Jilin Province and provincial colleges (SXGJQY2017-12), the Jilin Province Science and Technology Development Plan (20190304044YY), the Innovative Special Industry Fund Project in Jilin Province (2018C049-2), and the Open Research Project of the State Key Laboratory of Industrial Control Technology, Zhejiang University, China (ICT1800381). We would like to thank Editage [https://www.editage.cn] for the English language editing.

\section{References}

[1] S. J. Yu, H. J. Kim, E. S. Lee, C. G. Park, S. J. Cho, and S. H. Jeon, " $\beta$-Catenin accumulation is associated with increased expression of Nanog protein and predicts maintenance of
MSC self-renewal," Cell Transplantation, vol. 26, no. 2, pp. 365-377, 2017.

[2] S. Son, M. S. Liang, P. Lei, X. Xue, E. P. Furlani, and S. T. Andreadis, "Magnetofection mediated transient NANOG overexpression enhances proliferation and myogenic differentiation of human hair follicle derived mesenchymal stem cells," Bioconjugate Chemistry, vol. 26, no. 7, pp. 13141327, 2015.

[3] I. Perez-Estenaga, F. Prosper, and B. Pelacho, "Allogeneic mesenchymal stem cells and biomaterials: the perfect match for cardiac repair?," International Journal of Molecular Sciences, vol. 19, no. 10, article 3236, 2018.

[4] Y. H. Wang, D. B. Wu, B. Chen, E. Q. Chen, and H. Tang, "Progress in mesenchymal stem cell-based therapy for acute liver failure," Stem Cell Research \& Therapy, vol. 9, no. 1, p. 227, 2018.

[5] C. Kuppe and R. Kramann, "Role of mesenchymal stem cells in kidney injury and fibrosis," Current Opinion in Nephrology and Hypertension, vol. 25, no. 4, pp. 372-377, 2016.

[6] W. U. Hassan, U. Greiser, and W. Wang, "Role of adiposederived stem cells in wound healing," Wound Repair and Regeneration, vol. 22, no. 3, pp. 313-325, 2014.

[7] Y. Tang, Y. C. Cui, X. J. Wang et al., "Neural progenitor cells derived from adult bone marrow mesenchymal stem cells promote neuronal regeneration," Life Sciences, vol. 91, no. 19-20, pp. 951-958, 2012.

[8] J. Yu, M. A. Vodyanik, K. Smuga-Otto et al., "Induced pluripotent stem cell lines derived from human somatic cells," Science, vol. 318, no. 5858, pp. 1917-1920, 2007.

[9] C. C. Tsai, P. F. Su, Y. F. Huang, T. L. Yew, and S. C. Hung, "Oct4 and Nanog directly regulate Dnmt1 to maintain selfrenewal and undifferentiated state in mesenchymal stem cells," Molecular Cell, vol. 47, no. 2, pp. 169-182, 2012.

[10] A. Shahini, D. Choudhury, M. Asmani, R. Zhao, P. Lei, and S. T. Andreadis, "NANOG restores the impaired myogenic differentiation potential of skeletal myoblasts after multiple population doublings," Stem Cell Research, vol. 26, pp. 55-66, 2018.

[11] A. Shahini, P. Mistriotis, M. Asmani, R. Zhao, and S. T. Andreadis, "NANOG restores contractility of mesenchymal stem cell-based senescent microtissues," Tissue Engineering Part A, vol. 23, no. 11-12, pp. 535-545, 2017.

[12] J. Han, P. Mistriotis, P. Lei, D. Wang, S. Liu, and S. T. Andreadis, "Nanog reverses the effects of organismal aging on mesenchymal stem cell proliferation and myogenic differentiation potential," Stem Cells, vol. 30, no. 12, pp. 27462759, 2012.

[13] B. Münst, M. C. Thier, D. Winnemöller, M. Helfen, R. P. Thummer, and F. Edenhofer, "Nanog induces suppression of senescence through downregulation of $27^{\mathrm{KIP} 1}$ expression," Journal of Cell Science, vol. 129, no. 5, pp. 912-920, 2016.

[14] B. M. Grebbin, A. C. Hau, A. Groß et al., "Pbxl is required for adult subventricular zone neurogenesis," Development, vol. 143, no. 13, pp. 2281-2291, 2016.

[15] B. Xu, L. Cai, J. M. Butler et al., "The chromatin remodeler BPTF activates a stemness gene-expression program essential for the maintenance of adult hematopoietic stem cells," Stem Cell Reports, vol. 10, no. 3, pp. 675-683, 2018.

[16] W. Li, C. Y. Lin, C. Shang et al., "Pbx1 activates Fgf10 in the mesenchyme of developing lungs," Genesis, vol. 52, no. 5, pp. 399-407, 2014. 
[17] B. M. Grebbin and D. Schulte, "PBX1 as pioneer factor: a case still open," Frontiers in Cell and Development Biology, vol. 5, p. 9, 2017.

[18] C. L. Cheung, B. Y. Chan, V. Chan et al., "Pre-B-cell leukemia homeobox 1 (PBX1) shows functional and possible genetic association with bone mineral density variation," Human Molecular Genetics, vol. 18, no. 4, pp. 679-687, 2009.

[19] M. C. Monteiro, M. Sanyal, M. L. Cleary et al., "PBX1: a novel stage-specific regulator of adipocyte development," Stem Cells, vol. 29, no. 11, pp. 1837-1848, 2011.

[20] C. Li and J. Wang, "Quantifying cell fate decisions for differentiation and reprogramming of a human stem cell network: landscape and biological paths," PLoS Computational Biology, vol. 9, no. 8, article e1003165, 2013.

[21] K. K. Chan, J. Zhang, N. Y. Chia et al., "KLF4 and PBX1 directly regulate NANOG expression in human embryonic stem cells," Stem Cells, vol. 27, no. 9, pp. 2114-2125, 2009.

[22] L. A. Boyer, T. I. Lee, M. F. Cole et al., "Core transcriptional regulatory circuitry in human embryonic stem cells," Cell, vol. 122, no. 6, pp. 947-956, 2005.

[23] D. Piestun, B. S. Kochupurakkal, J. Jacob-Hirsch et al., "Nanog transforms NIH3T3 cells and targets cell-type restricted genes," Biochemical and Biophysical Research Communications, vol. 343, no. 1, pp. 279-285, 2006.

[24] S. Palumbo, T. L. Tsai, and W. J. Li, "Macrophage migration inhibitory factor regulates AKT signaling in hypoxic culture to modulate senescence of human mesenchymal stem cells," Stem Cells and Development, vol. 23, no. 8, pp. 852-865, 2014.

[25] X. Han, D. Liu, Y. Zhang et al., "Akt regulates TPP1 homodimerization and telomere protection," Aging Cell, vol. 12, no. 6, pp. 1091-1099, 2013.

[26] T. Bai, F. Liu, F. Zou et al., "Epidermal growth factor induces proliferation of hair follicle-derived mesenchymal stem cells through epidermal growth factor receptor-mediated activation of ERK and AKT signaling pathways associated with upregulation of Cyclin D1 and downregulation of p16," Stem Cells and Development, vol. 26, no. 2, pp. 113-122, 2017.

[27] S. Liu, S. Liu, X. Wang et al., "The PI3K-Akt pathway inhibits senescence and promotes self-renewal of human skinderived precursors in vitro," Aging Cell, vol. 10, no. 4, pp. 661-674, 2011.

[28] Y. Y. Kim, H. J. Jee, J. H. Um, Y. M. Kim, S. S. Bae, and J. Yun, "Cooperation between p21 and Akt is required for p53-dependent cellular senescence," Aging Cell, vol. 16, no. 5, pp. 1094$1103,2017$.

[29] X. Zhang, Y. Wang, Y. Gao et al., "Maintenance of high proliferation and multipotent potential of human hair follicle-derived mesenchymal stem cells by growth factors," International Journal of Molecular Medicine, vol. 31, no. 4, pp. 913-921, 2013.

[30] J. Y. Liu, H. F. Peng, S. Gopinath, J. Tian, and S. T. Andreadis, "Derivation of functional smooth muscle cells from multipotent human hair follicle Mesenchymal stem cells," Tissue Engineering Part A, vol. 16, no. 8, pp. 2553-2564, 2010.

[31] P. Li, F. Liu, C. Wu et al., "Feasibility of human hair folliclederived mesenchymal stem cells/CultiSpher $\left({ }^{\circledR}\right)-\mathrm{G}$ constructs in regenerative medicine," Cell and Tissue Research, vol. 362, no. 1, pp. 69-86, 2015.

[32] M. T. Kiani, C. A. Higgins, and B. D. Almquist, "The hair follicle: an underutilized source of cells and materials for regener- ative medicine," ACS Biomaterials Science \& Engineering, vol. 4, no. 4, pp. 1193-1207, 2018.

[33] Z. C. Xu, Q. Zhang, and H. Li, "Engineering of the human vessel wall with hair follicle stem cells in vitro," Molecular Medicine Reports, vol. 15, no. 1, pp. 417-422, 2017.

[34] C. Wu, F. Liu, P. Li et al., "Engineered hair follicle mesenchymal stem cells overexpressing controlled-release insulin reverse hyperglycemia in mice with type L diabetes," Cell Transplantation, vol. 24, no. 5, pp. 891-907, 2015.

[35] A. Kumar, S. Mohanty, K. Sahni, R. Kumar, and S. Gupta, "Extracted hair follicle outer root sheath cell suspension for pigment cell restoration in vitiligo," Journal of Cutaneous and Aesthetic Surgery, vol. 6, no. 2, pp. 121-125, 2013.

[36] B. Wang, W. Y.-W. Lee, B. Huang et al., "Secretome of human fetal mesenchymal stem cell ameliorates replicative senescence," Stem Cells and Development, vol. 25, no. 22, pp. 1755-1766, 2016.

[37] Y. Li and T. O. Tollefsbol, " $p 16^{I N K 4 a}$ suppression by glucose restriction contributes to human cellular lifespan extension through SIRT1-mediated epigenetic and genetic mechanisms," PLoS One, vol. 6, no. 2, article e17421, 2011.

[38] J. Kim, "Spermidine is protective against kidney ischemia and reperfusion injury through inhibiting DNA nitration and PARP1 activation," Anatomy \& Cell Biology, vol. 50, no. 3, pp. 200-206, 2017.

[39] G. A. Shilovsky, S. I. Shram, G. V. Morgunova, and A. N. Khokhlov, "Protein poly(ADP-ribosyl)ation system: changes in development and aging as well as due to restriction of cell proliferation," Biochemistry, vol. 82, no. 11, pp. 1391-1401, 2017.

[40] T. S. Piskunova, M. N. Yurova, A. I. Ovsyannikov et al., "Deficiency in poly(ADP-ribose) polymerase-1 (PARP-1) accelerates aging and spontaneous carcinogenesis in mice," Current Gerontology and Geriatrics Research, vol. 2008, Article ID 754190, 11 pages, 2008.

[41] T. Ogasawara, S. Ohba, F. Yano et al., "Nanog promotes osteogenic differentiation of the mouse mesenchymal cell line C3H10T1/2 by modulating bone morphogenetic protein (BMP) signaling," Journal of Cellular Physiology, vol. 228, no. 1, pp. 163-171, 2013.

[42] D. T. Chu and Y. Tao, "Molecular connections of obesity and aging: a focus on adipose protein 53 and retinoblastoma protein," Biogerontology, vol. 18, no. 3, pp. 321-332, 2017.

[43] L. Ho, L. Wang, T. M. Roth et al., "Sirtuin-3 promotes adipogenesis, osteoclastogenesis, and bone loss in aging male mice," Endocrinology, vol. 158, no. 9, pp. 2741-2753, 2017.

[44] F. Z. Asumda and P. B. Chase, "Age-related changes in rat bone-marrow mesenchymal stem cell plasticity," BMC Cell Biology, vol. 12, no. 1, p. 44, 2011.

[45] T. H. Ambrosi, A. Scialdone, A. Graja et al., "Adipocyte accumulation in the bone marrow during obesity and aging impairs stem cell-based hematopoietic and bone regeneration," Cell Stem Cell, vol. 20, no. 6, pp. 771-784.e6, 2017.

[46] H. D. Kim, C.-Y. Jang, J. M. Choe, J. Sohn, and J. Kim, "Phenylbutyric acid induces the cellular senescence through an $\mathrm{Akt} / \mathrm{p} 21^{\mathrm{WAF} 1}$ signaling pathway," Biochemical and Biophysical Research Communications, vol. 422, no. 2, pp. 213-218, 2012.

[47] Y. Li, D. Dowbenko, and L. A. Lasky, "AKT/PKB phosphorylation of $\mathrm{p} 21^{\mathrm{Cip} / \mathrm{WAF} 1}$ enhances protein stability of $\mathrm{p} 21^{\mathrm{Cip} / \mathrm{WAF} 1}$ and promotes cell survival," The Journal of Biological Chemistry, vol. 277, no. 13, pp. 11352-11361, 2002. 
[48] Y. Yu, D. Zhang, H. Huang et al., "NF- $\kappa$ B1 p50 promotes p53 protein translation through miR-190 downregulation of PHLPP1," Oncogene, vol. 33, no. 8, pp. 996-1005, 2014.

[49] Y. Jiang, F. Liu, F. Zou et al., "PBX homeobox 1 enhances hair follicle mesenchymal stem cell proliferation and reprogramming through activation of the AKT/glycogen synthase kinase signaling pathway and suppression of apoptosis," Stem Cell Research \&Therapy, vol. 10, no. 1, p. 268, 2019. 


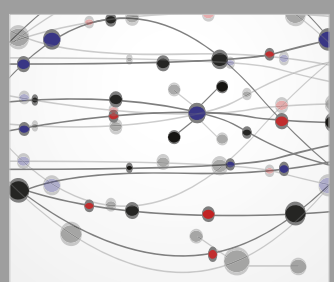

The Scientific World Journal
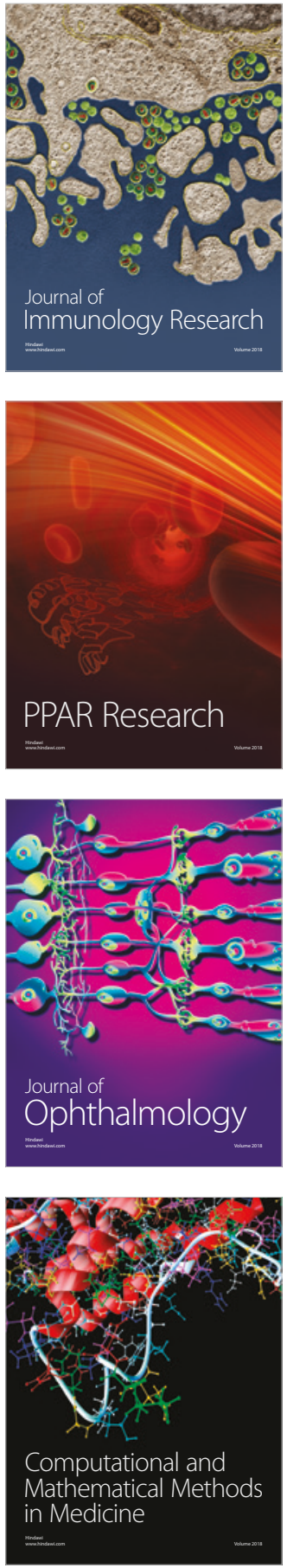

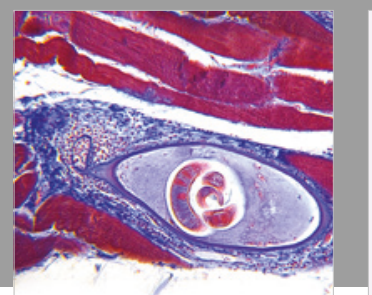

Gastroenterology Research and Practice

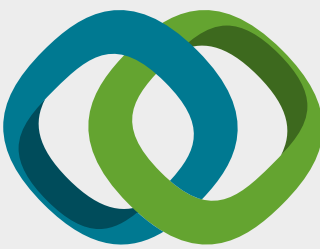

\section{Hindawi}

Submit your manuscripts at

www.hindawi.com
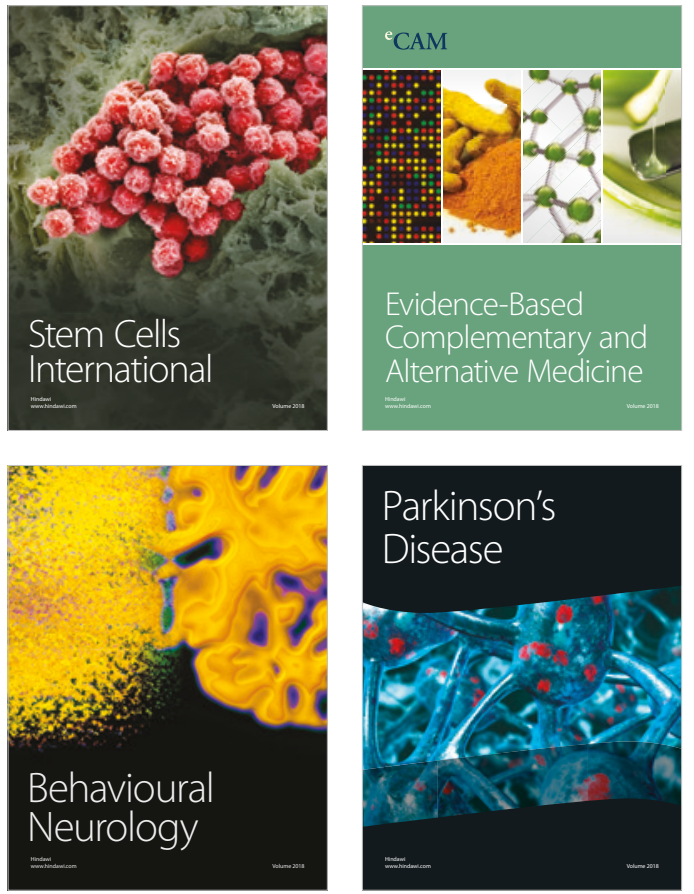

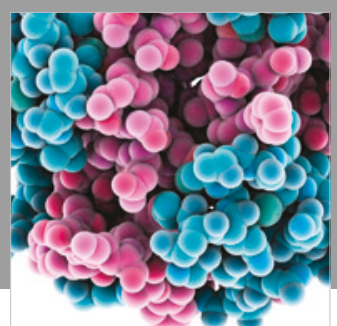

ournal of

Diabetes Research

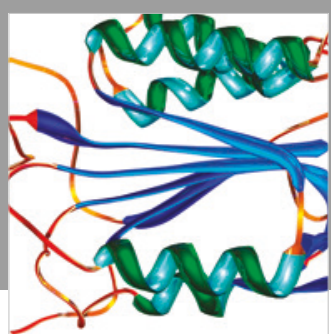

Disease Markers
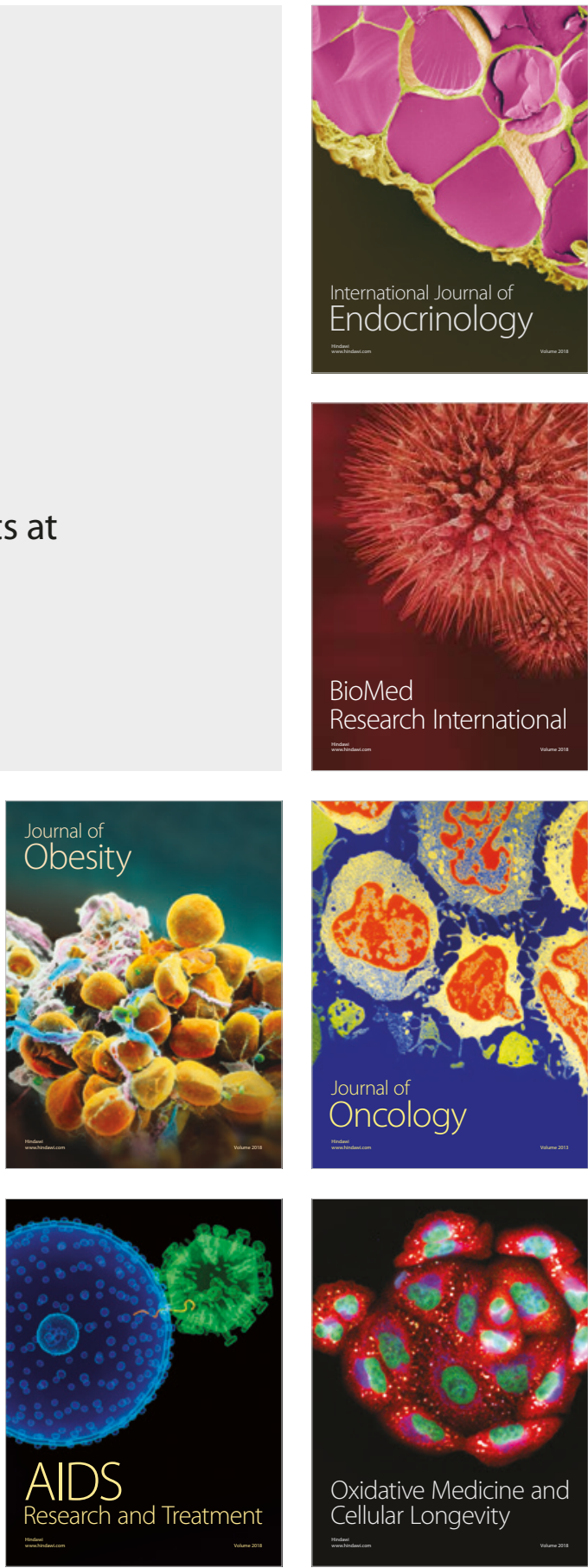